\title{
Production of hadrons with large transverse momentum in DIS at NLO *
}

\author{
Alejandro Daleo ${ }^{\dagger}$ \\ Institute for Theoretical Physics, University of Zuerich, Switzerland \\ E-mail: daleo@physik.unizh.ch
}

\section{Daniel de Florian}

Departamento de Física, Universidad de Buenos Aires, Ciudad Universitaria, Pab.1 (1428)

Buenos Aires, Argentina

E-mail: deflo@df.uba.ar

\section{Rodolfo Sassot}

Departamento de Física, Universidad de Buenos Aires, Ciudad Universitaria, Pab.1 (1428)

Buenos Aires, Argentina

E-mail: sassot@df.uba.ar

\begin{abstract}
We compute the order $\alpha_{s}^{2}$ corrections to the one particle inclusive electroproduction cross section of hadrons with non vanishing transverse momentum. We perform the full calculation analytically, and obtain the expression of the factorized (finite) cross section at this order. We compare our results with $\mathrm{H} 1$ data on forward production of $\pi^{0}$, and discuss the phenomenological implications of the rather large higher order contributions obtained in that case.
\end{abstract}

International Europhysics Conference on High Energy Physics

July 21st - 27th 2005

Lisboa, Portugal

*Partially supported by CONICET, Antorchas, UBACYT and ANPCyT, Argentina, and the Swiss National Science Foundation (SNF) through grants No. 200021-101874 and No. 200020-109162 and by the Forschungskredit der Universität Zürich..

†Speaker. 


\section{Introduction}

The study of the hadronic final state in deep inelastic scattering (DIS) provides an excellent opportunity to explore and test the main components of quantum chromodynamics: the structure of the hadronic initial state, the hadronization process and the higher order corrections in perturbation theory. Furthermore, both ZEUS and H1 experiments at HERA have produced many interesting and precise measurements of a variety of processes and these results are ever expanding and improving their accuracy. Some processes studied are, for example, different observables in jet production [1, 2], production of heavy flavors [3, 4] and semi-inclusive production of light hadrons [5],

The theoretical description of DIS processes beyond the totally inclusive one becomes more and more involved due to the delicate cancellation of singularities between real and virtual contributions. Recently there has been progress in the calculation of NLO corrections to the production of light hadrons with large transverse momentum $[6,7,8]$. In what follows we summarize the results obtained in the analytic calculation of [6].

\section{Outline of the calculation}

We consider the process

$$
l(l)+P(P) \longrightarrow l^{\prime}\left(l^{\prime}\right)+h\left(P_{h}\right)+X,
$$

where a lepton of momentum $l$ scatters off a nucleon of momentum $P$ with a lepton of momentum $l^{\prime}$ and a hadron $h$ of momentum $P_{h}$ tagged in the final state. Omitting target fragmentation, discussed at length in $[9,10]$ and that only contributes at zero transverse momentum, the cross section differential in transverse momentum and rapidity reads

$$
\frac{d \sigma^{h}}{d x_{B} d Q^{2} d p_{T}^{2} d \eta}=\sum_{i, j, n} \frac{e^{-\eta} \sqrt{S}}{\left|p_{T}\right|\left(Q^{2}+S\right)} \int_{e^{\eta} \frac{e^{2 p} T}{\sqrt{S}}}^{\frac{e^{2 \eta}}{1+e^{2 \eta}}} \frac{d y}{1-y} \int_{0}^{1-\frac{y}{1-y} e^{-2 \eta}} \frac{d z}{1-z} f_{i}(\xi) D_{h / j}(\zeta) \frac{d \sigma_{i j}^{(n)}}{d x_{B} d Q^{2} d y d z} .
$$

The partonic cross sections in Eq. (2.1) are calculated order by order in perturbation theory. For non vanishing transverse momentum, they start at order $\alpha_{s}$. The NLO corrections involve processes with two and three particles in the final state, corresponding to virtual and real corrections respectively. Both these contributions present ultraviolet and infrared singularities. The former are canceled by coupling constant renormalization. A subset of the latter are universally factorized in the redefinition of parton densities and fragmentation functions, whereas the remaining ones must cancel when combining real and virtual terms. This cancellation is only achieved after the integration over the loop momenta and the remaining phase space of the corresponding matrix elements. The resulting partonic cross sections are, thus, free of any remaining singularity and can be combined, as in Eq. (2.1), with PDFs and fragmentation functions in numerical programs to evaluate the corresponding cross sections.

\section{Comparison with experimental results}

In Fig. 1 we compare our results for the production of neutral pions as a function of $x_{B}$ with data obtained by the H1 experiment [5]. We show both the LO and NLO predictions, computed 
with MRST02 PDFs [11] and the fragmentation functions of references [12] and [13]. The renormalization and factorization scales have been fixed, $\mu^{2}=\left(Q^{2}+p_{T}^{2}\right) / 2$. On the right panel we plot the uncertainty of the NLO calculation due to the variation of these scales by a factor of 2 .. The
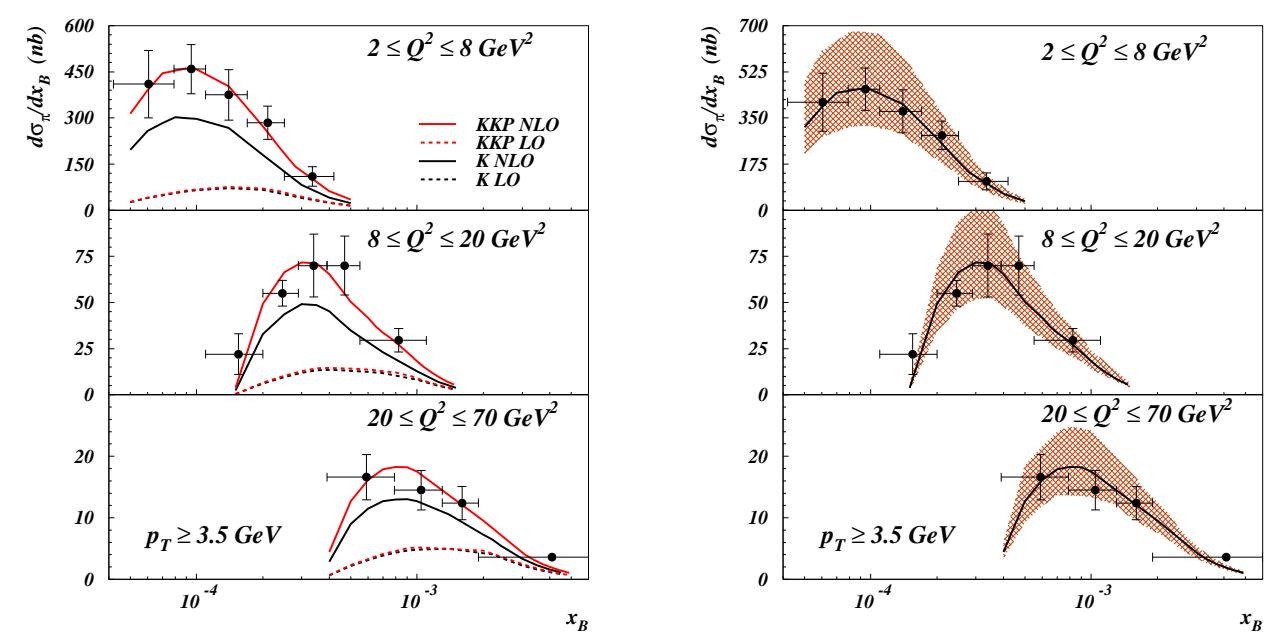

Figure 1: Results for the $x_{B}$ distribution at NLO including the scale uncertainty.

NLO calculation reproduces fairly well the data. Strikingly, the corrections are several times larger that the LO contributions. Also the scale uncertainty is quite noticeable. These effects have been also observed in other processes in photon induced collisions [14, 15].

The rather large size of the NLO corrections can be understood as a consequence of the opening of a new dominant ('leading-order') channel, and not to the 'genuine' increase in the partonic
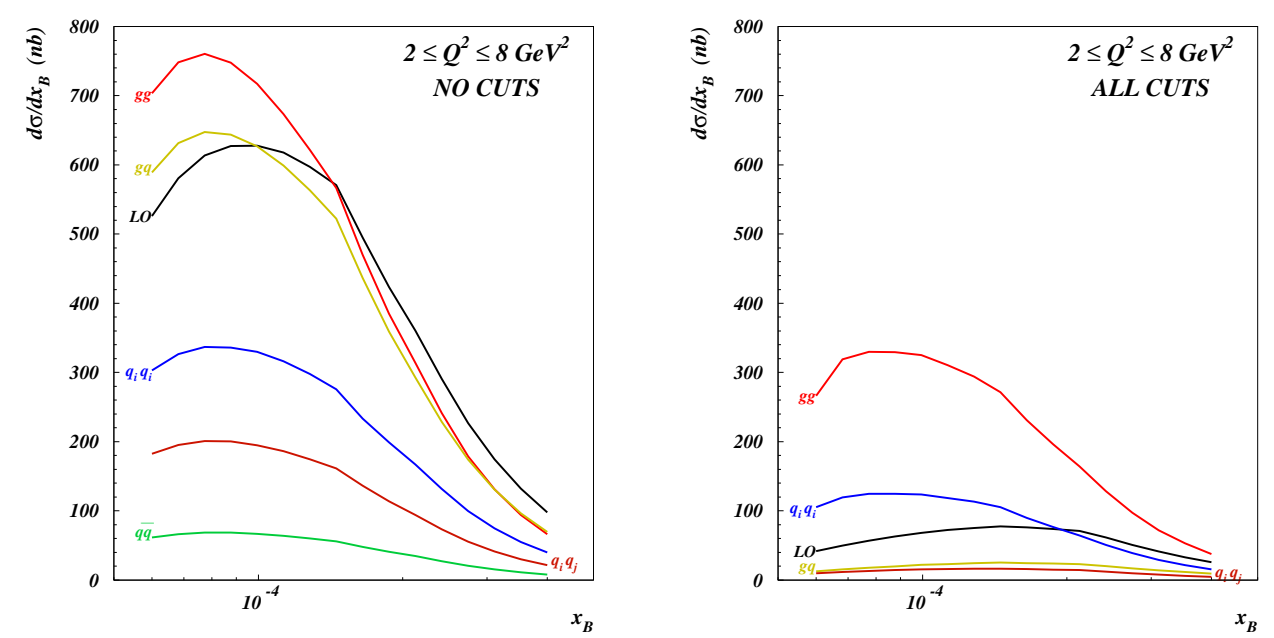

Figure 2: The $x_{B}$ distribution discriminated by partonic process without and with the experimental cuts.

cross section that might otherwise threaten perturbative stability. The dominance of the new channel is due to the size of the gluon distribution at small $x_{B}$ and to the fact that the H1 selection cuts highlight the kinematical region dominated by the $\gamma+g \rightarrow g+q+{ }^{-} q$ partonic process, as shown in 
Fig. 2. In particular, without the experimental cuts for the final state hadrons, the $g g$ component represents less than $25 \%$ of the total NLO contribution at small $x_{B}$, which is dominated by the $g q$ subprocess. The forward selection is also responsible of the scale sensitivity of the cross section, as it suppresses large components with small scale dependence whereas it stresses components as $g g$ whose scale dependence would be partly canceled only at NNLO.

\section{Conclusions}

We have presented the analytical calculation of the differential cross section for semi-inclusive production of a hadron, with non vanishing transverse momentum, in DIS at next-to-leading-order in QCD. We found that the order $\alpha_{s}^{2}$ corrections are important. We compared our results with recent data coming from the $\mathrm{H} 1$ experiment at HERA. Within the uncertainties arising from the scale dependence and the particular sensitivity of the results to the gluon hadronization mechanism, parameterized in the fragmentation functions, we found a very good agreement between data and theoretical expectations for both the $x_{B}$ and $p_{T}$ distributions. We also found that the experimental cuts applied to the $\mathrm{H} 1$ data play a crucial role, boosting the NLO corrections. The main contributions to these corrections come from the partonic subprocess $\gamma+g \rightarrow g+q+{ }^{-} q$ which appears for the first time at that order. The appearance of new channels also leads to quite a significant factorization scale dependence even at the NLO level. Thus, after the experimental cuts, the contributions of order $\alpha_{s}^{2}$ effectively resemble a LO description.

\section{References}

[1] S. Chekanov et al. [ZEUS Collaboration], arXiv:hep-ex/0502007.

[2] A. Aktas et al. [H1 Collaboration], arXiv:hep-ex/0508055.

[3] A. Aktas et al. [H1 Collaboration], Eur. Phys. J. C 41, 453 (2005) [arXiv:hep-ex/0502010].

[4] S. Chekanov et al. [ZEUS Collaboration], Phys. Rev. D 69, 012004 (2004) [arXiv:hep-ex/0308068].

[5] A. Aktas et al. [H1 Collaboration], Eur. Phys. J. C 36, 441 (2004) [arXiv:hep-ex/0404009].

[6] A. Daleo, D. de Florian and R. Sassot, Phys. Rev. D 71, 034013 (2005) [arXiv:hep-ph/0411212].

[7] P. Aurenche, R. Basu, M. Fontannaz and R. M. Godbole, Eur. Phys. J. C 34, 277 (2004) [arXiv:hep-ph/0312359].

[8] B. A. Kniehl, G. Kramer and M. Maniatis, Nucl. Phys. B 711, 345 (2005) [Erratum-ibid. B 720, 345 (2005)] [arXiv:hep-ph/0411300].

[9] A. Daleo, C. A. Garcia Canal and R. Sassot, Nucl. Phys. B 662, 334 (2003) [arXiv:hep-ph/0303199].

[10] A. Daleo and R. Sassot, Nucl. Phys. B 673, 357 (2003) [arXiv:hep-ph/0309073].

[11] A. D. Martin, R. G. Roberts, W. J. Stirling and R. S. Thorne, Eur. Phys. J. C 28, 455 (2003) [arXiv:hep-ph/0211080].

[12] B. A. Kniehl, G. Kramer and B. Potter, Nucl. Phys. B 582, 514 (2000) [arXiv:hep-ph/0010289].

[13] S. Kretzer, Phys. Rev. D 62, 054001 (2000) [arXiv:hep-ph/0003177].

[14] J. Chyla, arXiv:hep-ph/0511132.

[15] A. Daleo and R. Sassot, arXiv:hep-ph/0511189. 